\title{
ANÁLISIS CITOLÓGICO DE UNA POBLACIÓN DE Bomarea salsilla (L.) MIRB. (ALSTROEMERIACEAE) DE LA REGIÓN DEL BIOBÍO, CHILE
}

\section{CYTOLOGICAL ANALYSIS IN A POPULATION OF Bomarea salsilla (L.) MIRB. (ALSTROEMERIACEAE) FROM BIOBIO REGION, CHILE}

\author{
Carlos Baeza ${ }^{1 *}$, y Rosa Silva ${ }^{2}$ \\ ${ }^{1}$ Facultad de Ciencias Naturales y Oceanográficas, Departamento de Botánica, Universidad de Concep- \\ ción, Casilla 2407, Concepción, Chile \\ 2 Escuela de Ciencias y Tecnología, Universidad de Concepción, Casilla 341, Los Angeles \\ * Autor para correspondencia E-mail: cbaeza@udec.cl
}

\section{RESUMEN}

Bomarea salsilla (L.) Mirb. es una especie endémica de Chile, de la familia Alstroemeriaceae, de amplia distribución en el país y de enorme potencial ornamental. En este trabajo se describe el cariotipo de una población de Bomarea salsilla de la Región de Biobío, Chile. Se determinó un cariotipo $2 n=2 x=18$ y una fórmula haploide de $2 \mathrm{~m}+1 \mathrm{sm}+3 \mathrm{st}+2 \mathrm{st}$-sat $+1 \mathrm{t}$-sat. Se compararon estos resultados con los de otra población de la misma especie ya publicados, pero de la Región de Valparaíso. Se concluyó que ambas poblaciones presentan un cariotipo e índices de asimetría prácticamente iguales, lo que refuerza la idea que el cariotipo es muy estable dentro de algunas especies, independiente de su distribución geográfica.

Palabras clave: Alstroemeriaceae, B. salsilla, cariotipo, Chile.

\begin{abstract}
Bomarea salsilla (L.) Mirb. is an endemic species of Chile that belongs to the family Alstroemeriaceae. It is widely distributed throughout the country and it has a high ornamental potential. This study describes the karyotype of a population of this species from Biobio Region, Chile. A karyotype $2 \mathrm{n}=2 \mathrm{x}=18$, with a haploid formula of $2 \mathrm{~m}+1 \mathrm{sm}+3 \mathrm{st}+2 \mathrm{st}-\mathrm{sat}+1 \mathrm{t}$-sat, was determined. These results were compared with those previously published for other population of the same species but from Valparaiso Region. It was concluded that both populations have practically the same karyotype and asymmetry indices, which reinforces the idea that the karyotype is very stable within some species, regardless of their geographical distribution.
\end{abstract}

Key words: Alstroemeriaceae, B. salsilla, karyotype, Chile. 


\section{INTRODUCCIÓN}

La familia Alstroemeriaceae es endémica de las regiones tropicales y templadas cálidas de América, desde México a Chile, presentando su mayor diversidad en los Andes. Está conformada por 3 géneros: Alstroemeria L., Bomarea Mirb. y Leontochir Phil. (Muñoz y Moreira, 2003). Según Muñoz y Moreira (2003) comprende alrededor de 183 taxones; el número de especies de Bomarea en Chile corresponde a tres: Bomarea involucrosa (Herb.) Baker, B. engleriana Kraenzl. y B. salsilla (L.) Mirb. Algunos autores (Hofreiter y Lyshede, 2006; Palma-Rojas et al., 2007; Alzate et al., 2008) consideran que Leontochir ovallei Phil. corresponde a una especie de Bomarea (Bomarea ovallei (Phil.) Ravenna). Sin embargo, otros autores consideran que Leontochir presenta características morfológicas y citológicas suficientes para considerarlo un género chileno monotípico válido (Baeza et al., 2012).

Bomarea salsilla es una especie trepadora, que posee tallos alargados de hasta $1 \mathrm{~m}$ de largo, raíces fibrosas con 7 a 9 tubérculos, con hojas lanceoladas u oblongo-lanceoladas de hasta $10 \mathrm{~cm}$, que tienen 5 nervios notorios, con pecíolos resupinados de $1 \mathrm{~cm}$, con flores dispuestas en umbela con 4-8 radios dicotómicos los cuales pueden medir hasta 7,5 cm, y tener 1-3 flores de color que varía del rosado oscuro al púrpura (Marticorena et al., 2010). Es una especie endémica de Chile, y la más austral del género Bomarea.

Sanso y Hunziker (1998) caracterizan el cariotipo de B. boliviensis Baker y B. edulis (Tussac) Herb., ambas de Argentina, indicando un $2 n=18$ cromosomas, y con la característica que el cromosoma 5 siempre es metacéntrico. Palma-Rojas et al. (2007) hicieron un estudio citológico en especies chilenas de Bomarea y en Leontochir ovallei, indicando que esta última especie comparte características del cariotipo muy similares con Bomarea, sugiriendo una relación filogenética estrecha entre ellas. Baeza et al. (2008) describen el cariotipo de Bomarea patinii Baker ssp. patinii, señalando que el cromosoma 5 es metacéntrico, lo que se mantiene como constante en las otras especies de Bomarea estudiadas.

Este trabajo tiene como objetivo evaluar si existe variación o estabilidad inter-poblacional o intra-específica en la morfología del cariotipo de la especie en estudio a lo largo de su distribución geográfica, entregando antecedentes cariológicos de una población de Bomarea salsilla colectada en la Región del Biobío, y compararla con otra población ya analizada citológicamente, pero de la Región de Valparaíso.

\section{MATERIALES Y MÉTODOS}

\section{Material vegetal}

El material vegetal usado en el estudio se obtuvo en la Comuna San Pedro de La Paz, Villa El Recodo ( $36^{\circ} 51^{\prime}$ S, $73^{\circ} 04^{\prime} \mathrm{W}$ ), provincia de Concepción, Región del Biobío, km. 2,5 camino a Santa Juana, en cerros aledaños a la Villa. El código de la colecta fue 31-XII-2011. C. Baeza 4353 $\mathrm{A}(\mathrm{CONC})$.

\section{Metodología}

El estudio cariológico se realizó siguiendo la metodología propuesta por Baeza et al. (2009): raíces (1-2 cm de longitud) obtenidas a partir de material cultivado en invernadero fueron cortadas y pretratadas con una solución de 8-hidroxiquinolina $(2 \mathrm{mM})$ durante 24 horas a $4^{\circ} \mathrm{C}$. Posteriormente, se fijaron en una mezcla fresca de etanol/ácido acético (3:1) por 24 horas. Se realizaron aplastados de puntas de raíces realizando previamente una hidrólisis ácida con $\mathrm{HCl} 0,5$ $\mathrm{N}$ durante 17 minutos a $42^{\circ} \mathrm{C}$, para luego lavar el material y teñir la punta de raíz con orceína al $1 \%$. Los cromosomas fueron fotografiados en un microscopio Zeiss Axioskop con cámara de video incorporada. Las placas metafásicas fueron medidas con la ayuda del programa computacional "MicroMeasure 3.3" (Reeves 2001). Para la población analizada (20 placas metafásicas) se determinaron los índices Ask\% de Arano y Saito (1980), Syi de Greilhuber y Speta (1976), los índices $A_{1}$ y $\mathrm{A}_{2}$ de Romero Zarco (1986), los coeficientes $\mathrm{CV}_{\mathrm{CI}}$ y $\mathrm{CV}_{\mathrm{CL}}$ de Paszko (2006) y el índice $\mathrm{M}_{\mathrm{CA}}$ (Peruzzi y Eroğlu, 2013). También se calculó la longitud total diploide (LTC) de los cromosomas expresada en micrones. Los cromosomas se clasificaron de acuerdo a la nomenclatura de Levan et al. (1964).

\section{RESULTADOS Y DISCUSIÓN}

La población analizada presenta un número diploide $2 n=18$ cromosomas, con una fórmula haploide $2 \mathrm{~m}+1 \mathrm{sm}+3 \mathrm{st}+2 \mathrm{st}$-sat $+1 \mathrm{t}$-sat, esto es, dos cromosomas metacéntricos (pares 1 y 5), un par submetacéntrico (par 9, a veces presenta un microsatélite en el brazo corto), tres pares subtelocéntricos (pares 4, 6 y 8), dos pares subtelocéntricos con un microsatélite en el brazo corto (pares 3 y 7) y un par de cromosomas telocéntricos con satélite (par 2). (Tabla 1; Figs. 1 y 2).

Al comparar los cromosomas encontrados en la población analizada, en relación a lo reportado por Palma-Rojas et al. (2007), se encontraron diferencias mínimas entre ellos, esto es, la presencia de microsatélites en los brazos cortos de los cromosomas dos y siete, situación que no aparece en la población de la Región de Valparaíso anali- 
Tabla 1. Mediciones cromosómicas de Bomarea salsilla (L.) Mirb., con longitudes promedio como porcentaje de la longitud del genoma haploide de 20 metafases.

Table 1. Chromosomal measurements of Bomarea salsilla (L.) Mirb. with mean length as percent of the haploid genome length of 20 metaphases.

\begin{tabular}{cccccc}
\hline $\begin{array}{c}\text { Par } \\
\text { cromosómico }\end{array}$ & $\begin{array}{c}\text { Long. brazo } \\
\text { largo }\end{array}$ & $\begin{array}{c}\text { Long. brazo } \\
\text { corto }\end{array}$ & $\begin{array}{c}\text { Long. total } \\
\text { relativa }\end{array}$ & $\begin{array}{c}\text { Long. total } \\
\text { absoluta }\end{array}$ & $\begin{array}{c}\text { Tipo de } \\
\text { cromosoma }^{1}\end{array}$ \\
\hline & $\% \pm$ D.S. & $\% \pm$ D.S. & $\%$ & $\mu \mathrm{m}$ & \\
1 & $4,30 \pm 0,38$ & $3,84 \pm 0,31$ & 8,14 & 8,52 & $1,11 \mathrm{~m}$ \\
2 & $6,76 \pm 0,70$ & $0,85 \pm 0,15$ & 7,61 & 8,01 & 8,06 t-sat \\
3 & $5,65 \pm 0,29$ & $1,00 \pm 0,17$ & 6,65 & 7,03 & 5,78 st-sat \\
4 & $4,76 \pm 0,51$ & $1,15 \pm 0,21$ & 5,91 & 6,20 & 4,21 st \\
5 & $2,96 \pm 0,76$ & $2,32 \pm 0,13$ & 5,28 & 5,54 & $1,28 \mathrm{~m}$ \\
6 & $4,07 \pm 0,35$ & $0,99 \pm 0,25$ & 5,06 & 5,23 & 4,11 st \\
7 & $3,85 \pm 0,20$ & $1,00 \pm 0,17$ & 4,85 & 5,10 & 3,93 st-sat \\
8 & $3,44 \pm 0,37$ & $0,99 \pm 0,21$ & 4,48 & 4,67 & 3,59 st \\
9 & $2,79 \pm 0,28$ & $1,21 \pm 0,18$ & 3,92 & 4,11 & 2,32 sm \\
\hline
\end{tabular}

${ }^{1} \mathrm{~m}$ = metacéntrico; $\mathrm{sm}$ = submetacéntrico; st = subtelocéntrico; $\mathrm{t}$ = telocéntrico; sat = presencia de microsatélites .

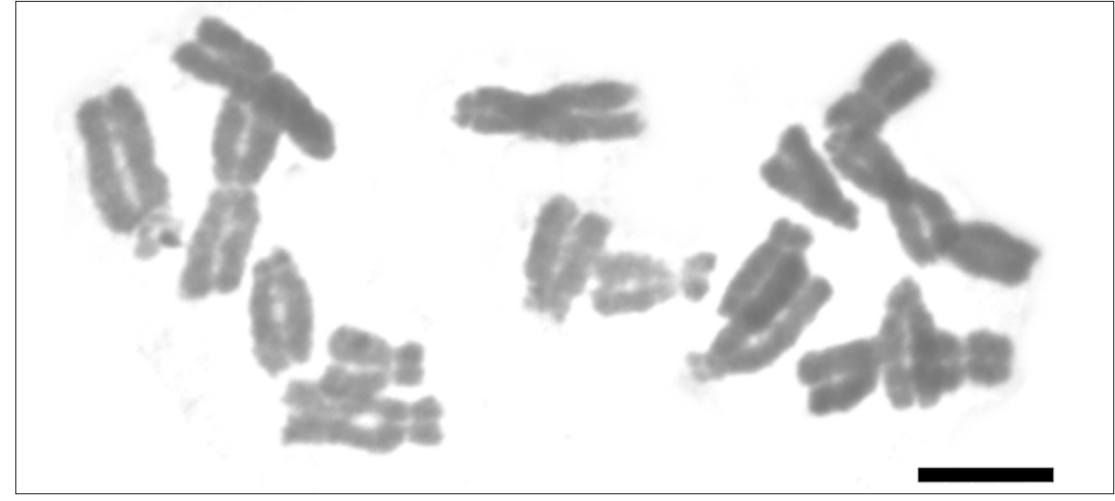

Fig. 1. Placa metafásica de Bomarea salsilla (L.) Mirb.

La escala corresponde a $5 \mu \mathrm{m}$.

Fig. 1. Methaphase chromosomes of Bomarea salsilla (L.) Mirb.

The scale corresponds to $5 \mu \mathrm{m}$.

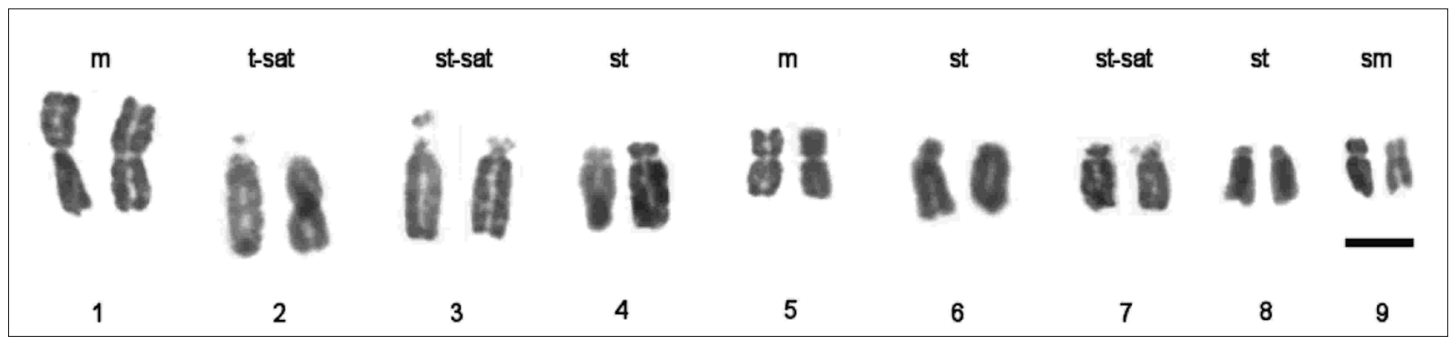

Fig. 2. Cariotipo de Bomarea salsilla (L.) Mirb. obtenido del análisis de 20 placas metafásicas.

La escala corresponde a $5 \mu \mathrm{m} . \mathrm{m}$ = metacéntrico; $\mathrm{sm}$ = submetacéntrico; $\mathrm{st}=$ subtelocéntrico; $\mathrm{t}$ = telocéntrico; sat $=$ presencia de microsatélites.

Fig. 2. Karyotype of Bomarea salsilla (L.) Mirb. from 20 methaphasic plates.

Scale correspons to $5 \mu \mathrm{m} . \mathrm{m}=$ metacentric; $\mathrm{sm}=$ submetacentric; $\mathrm{st}=$ subtelocentric; $\mathrm{t}=$ telocentric; sat $=$ with microsatellites. 
zada por Palma-Rojas et al. (2007). El resto de los cromosomas es idéntico en ambas poblaciones. Respecto a los índices de asimetría analizados y comparados en las dos poblaciones, no se observan diferencias significativas en ninguno de ellos (Tabla 2). Esta situación observada demuestra la enorme estabilidad en el cariotipo de algunas especies, independiente de su distribución geográfica. Algo muy similar también ha sido reportado para Alstroemeria hookeri Lodd. y Alstroemeria presliana Herbert (Baeza et al., 2010; Baeza et al. 2015). Esta condición de estabilidad del cariotipo está corroborando la importancia de los estudios citotaxonómicos y cariológicos como un complemento a la sistemática vegetal. La única diferencia observada en las poblaciones es la longitud total de los cromosomas (Tabla 2), cuyo tamaño es mayor en la población de la Región del Biobío. Esto puede deberse a que la colchicina, como agente inhibidor del huso acromático, tiende a contraer más los cromosomas que otras sustancias, por ejemplo 8-hidroxiquinoleina, lo que se traduce en un menor tamaño de ellos (Delgado et al., 2010).

\section{CONCLUSIONES}

Bomarea salsilla es una especie de la familia Alstroemeriaceae que presenta una alta estabilidad en la arquitectura de su cariotipo, no solo en cuanto a su morfología, sino también en cuanto a su biometría. Esto queda demostrado al analizar cualitativamente los cariotipos de ambas poblaciones como también cuantitativamente mediante los índices de asimetría utilizados. Además, al comparar el cariotipo de ambas poblaciones de $B$. salsilla con otras especies de Bomarea ya analizadas citológicamente, se mantiene la estabilidad del cariotipo lo que corrobora la gran estabilidad del cariotipo a nivel genérico.

\section{RECONOCIMIENTOS}

Se agradece al Departamento de Botánica y a la Dirección de Investigación de la Universidad de Concepción por las facilidades otorgadas.

\section{LITERATURA CITADA}

Alzate, F., M. Mort, and M. Ramírez. 2008. Phylogenetic analyses of Bomarea (Alstroemeriaceae) based on combined analysed of nrDNA ITS, psbA-trnH, rpob-trnC and matK sequences. Taxon 57(3):853-862.

Arano, H., and H. Saito. 1980. Cytological studies in family Umbelliferae 5. Karyotypes of seven species in subtribe Seselinae. La Kromosomo 2:471-480.

Baeza, C., F. Alzate, M. Negritto, y E. Ruiz. 2008. El cariotipo de Bomarea patinii Baker subsp. patinii (Alstroemeriaceae). Gayana Bot. 65(2):233-236.

Baeza, M., E. Ruiz, y M. Negritto. 2009. Importancia del cariotipo en la taxonomía y evolución del género Chaetanthera (Asteraceae): evidencias preliminares para especies que crecen en Chile. Gayana Bot. 66(1):50-57.

Baeza, C., E. Ruiz, y M. Rosas. 2012. Es Leontochir ovallei Phil. (Alstroemeriaceae) un género distinto a Bomarea Mirbel? Consideraciones citológicas. Agro-Ciencia Chile 28(1):63-67.

Baeza, C., E. Ruiz, and M. Negritto 2010. Comparative karyotypic analysis in the Alstroemeria hookeri Lodd. (Alstroemeriaceae) complex sensu Bayer (1987). Genet. Mol. Biol. 33(1):119-124.

Baeza, C., L. Finot, and E. Ruiz. 2015. Comparative karyotype analysis of populations in the Alstroemeria presliana Herbert (Alstroemeriaceae) complex in Chile. Genet. Mol. Biol. 38(2):199-204.

Tabla 2. Comparación de las dos poblaciones de Bomarea salsilla (L.) Mirb. analizadas de acuerdo a los índices de asimetría utilizados.

Table 2. Comparison of two populations of Bomarea salsilla (L.) Mirb analyzed according to the asymmetry indices used.

\begin{tabular}{lcc}
\hline Índices & B. salsilla $($ Baeza $4353 \mathbf{A})$ & B. salsilla (Palma et al., 2007) \\
\hline $\mathrm{A}_{1}$ & $0,62 \pm 0,02$ & $0,63 \pm 0,03$ \\
$\mathrm{~A}_{2}$ & $0,26 \pm 0,02$ & $0,28 \pm 0,02$ \\
Ask, $\%$ & $74,42 \pm 0,86$ & 73,49 \\
Syi & $34,38 \pm 1,55$ & 36,07 \\
$\mathrm{Cv}_{\mathrm{Cl}}$ & $49,63 \pm 4,47$ & 47,99 \\
$\mathrm{CV}_{\mathrm{CL}}$ & $25,63 \pm 1,52$ & 23,19 \\
$\mathrm{M}_{\mathrm{CA}}(\mu \mathrm{m})$ & $0,49 \pm 0,02$ & 0,48 \\
LTC $(\mu \mathrm{m})$ & 85 \\
\hline
\end{tabular}


Delgado, I., M. Uribe, y M. Marulanda. 2010. Estandarización de la técnica citogenética "Squash" para conteo de cromosomas mitóticos en Rubus glaucus Benth. Sci. Tech. 3(46):74-79.

Greilhuber, J., and F. Speta. 1976. C-banded karyotypes in the Scilla hohenackeri group, S. persica, and Puschkinia (Liliaceae). Plant Syst. Evol. 126:149-188.

Hofreiter, A., and O. Lyshede. 2006. Functional leaf anatomy of Bomarea Mirb. (Alstroemeriaceae). Bot. J. Linn. Soc. 152:73-90.

Levan, A, K. Fredga, and A. Sandberg. 1964. Nomenclature for centromeric position on chromosomes. Hereditas 52:201-220.

Marticorena, A., D. Alarcón, I. Abello, y C. Atala. 2010. Plantas trepadoras, epífitas y parásitas nativas de Chile. Guía de Campo. 291 p. Corporación de la Madera, Concepción, Chile.

Muñoz, M., y A. Moreira. 2003. Alstroemerias de Chile; Diversidad, distribución y conservación. 139 p. Taller La Era, Santiago, Chile.
Palma-Rojas, C., P. Jara-Seguel, and E. Von Brand. 2007. Karyological studies in Chilean species of Bomarea and Leontochir (Alstroemeriaceae). New Zealand J. Bot. 45:299-303.

Paszko, B. 2006. A critical review and a new proposal of karyotype asymmetry indices. Plant Syst. Evol. 258:39-48.

Peruzzi, L., and H. Eroğlu. 2013. Karyotype asymmetry: again, how to measure and what to measure?. CompCytogen 7(1):1-9.

Reeves, A. 2001. MicroMeasure: a new computer program for the collection and analysis of cytogenetic data. Genome 44:239-443.

Romero Zarco, C. 1986. A new method for estimating karyotype asymmetry. Taxon 35:526530.

Sanso, A., and J. Hunziker. 1998. Karyological studies in Alstroemeria and Bomarea (Alstroemeriaceae). Hereditas 129:67-74. 